\title{
Studying the influence of substitutional elements on mechanical behavior of Alloy 718
}

\author{
Bertrand Max ${ }^{1, \text { a }}$, Jose San Juan ${ }^{2}$, Maria L. Nó ${ }^{2}$, Jean-Marc Cloué ${ }^{1}$, Bernard Viguier ${ }^{1}$, and Eric Andrieu ${ }^{1}$ \\ ${ }^{1}$ CIRIMAT, INP, Université de Toulouse, 4 allée Emile Monso, 31030 Toulouse, France \\ ${ }^{2}$ Facultad de Ciencia y Tecnología, Universidad del País Vasco, Apdo. 644, 48080 Bilbao, Spain
}

\begin{abstract}
In nickel-based superalloys, substitutional solute species have a strong impact on in service mechanical properties as well as on oxidation and corrosion resistances. In alloy 718 , recent studies carried out by tensile tests highlighted the fact that refractory solute species are able to interact strongly with mobile dislocations during plastic deformation, generating dynamic strain ageing, and, in wide ranges of tests temperatures and strain rates, Portevin-Le Chatelier effect. The precise nature of the substitutional element responsible for such a dynamic interaction is still subject to debate. We addressed this question by means of mechanical spectroscopy studies of alloy 718 and various related alloys corresponding to monitored changes in the chemical composition. Only a single internal friction relaxation peak has been observed for all the studied alloys. By analyzing the damping behavior of these alloys at different imposed solicitation frequencies by sweeping a large temperatures range, the activation energies of the relaxation process and the type of mechanism involved have been determined. The process is a "Zener relaxation" in the alloys, i.e. a substitutional atoms dipole reorientation under applied stress. The results tend to prove that Niobium is not involved in the relaxation process whereas Molybdenum content seems to play an important role in the relaxation intensity.
\end{abstract}

\section{Introduction}

Alloy 718 is a widely used superalloy for high performance applications because of its excellent mechanical properties over a wide range of temperatures, as well as good oxidation and corrosion resistances. Substitutional alloying elements have a strong influence on these properties of the material: they are involved in solid solution hardening of the matrix, in the formation of strengthening phases and confer to the alloy its resistance to oxidation and corrosion [1-6]. Nevertheless, alloy 718 remains sensitive to intergranular stress corrosion cracking for various in service conditions for different types of applications.

During a study on the effect of oxidation on mechanical behavior of alloy 718, Fournier et al. [7] shown that the occurrence of Portevin-Le Chatelier (PLC) effect during tensile test systematically resulted in an absence of brittle area on the fracture surface of the samples. By contrast, for higher testing temperatures, when the stress-strain curves are smooth, intergranular cracking areas are always observed on the fracture surface. This observation was later confirmed by Garat [8]: the sharp transition boundary that exists between these fracture modes is superimposed with the boundary between deformation modes. The first explanations for such an observation considered only the mechanical aspects of the PLC phenomenon. However, the coupling between chemical aspects and mechanical behavior can also be considered: when the flow stress is stable, a dragging phenomenon of solute

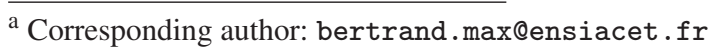

elements by mobile dislocations can occur continuously, leading to an enrichment of grain boundaries by the transported elements which may make grain boundaries more sensitive to oxidation phenomena. Thus, the study of dynamic interactions between solute element and mobile dislocations is a good way to access to information about which element is transported during plastic deformation.

In the past few decades, many studies [9-12] were conducted by means of tensile tests in order the study the dislocation - solutes interaction mechanism and try to identify the atomic species involved in such mechanism. The observation was made that in alloy 718 , dynamic strain ageing (DSA) phenomenon exists over wide ranges of temperature and strain rate, which include the occurrence of plastic instabilities known as PLC effect. Moreover, various regimes in the PLC domain were identified which have been attributed to the interaction of mobile dislocations with different alloying elements. Hence, in the domain of lower temperatures, the PLC effect is often considered to be due to the dynamic interaction between carbon atoms and mobile dislocations, while in the domain of high temperatures, the PLC effect is considered as a result of the interaction of a slower diffusing species, namely a substitutional one. Nevertheless, no consensus was reached regarding its exact nature: some authors [11] consider $\mathrm{Cr}$ as potentially responsible for high temperature PLC effect, while for other authors [9, 12], the explanation lies in solid solution $\mathrm{Nb}$ content of the alloy. A common way to try identifying the solute species involved in the occurrence of the PLC effects is to compare the calculated 


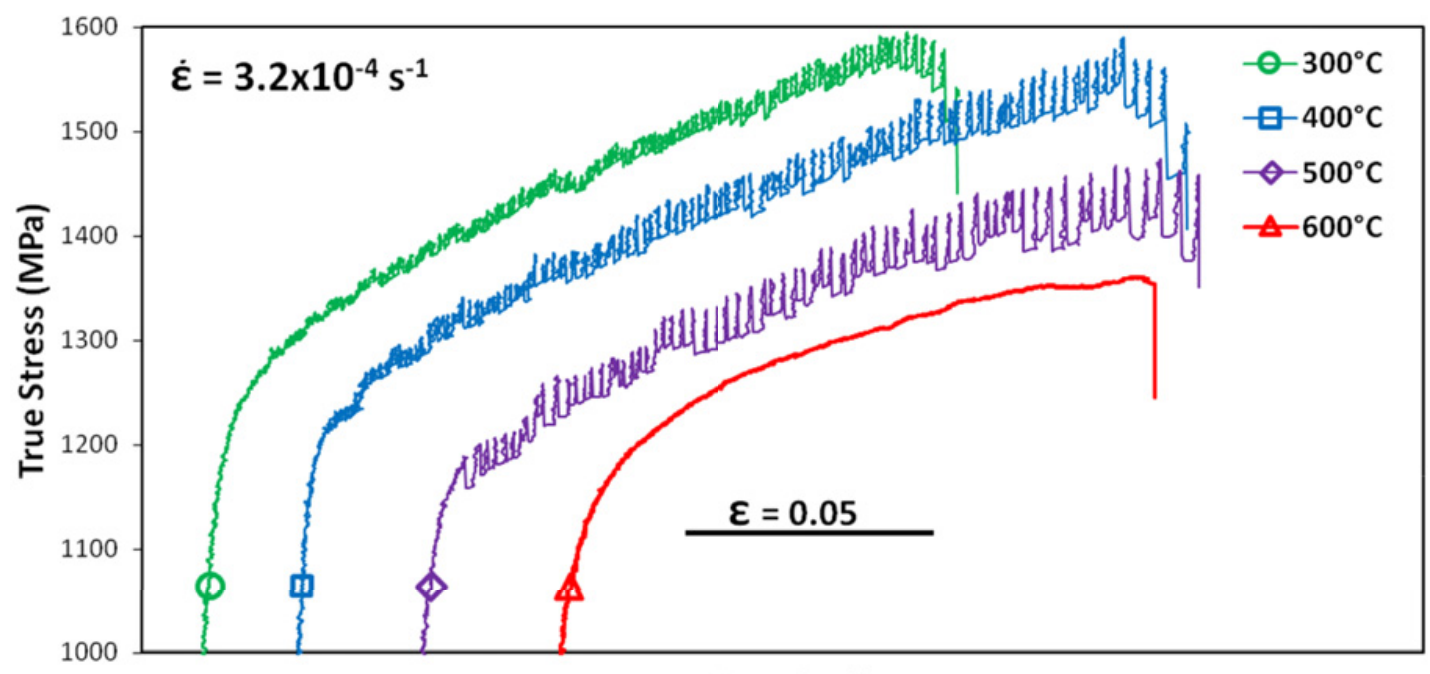

True Strain

Figure 1. True stress-true strain tensile curves obtained at $\varepsilon=3.210^{-4} \mathrm{~s}^{-1}$ on heat treated alloy 718 for various temperatures and showing different serration behavior. The curves are offset from each other for better visualization.

apparent activation energies with the activation energies for de diffusion of various solute species in the alloy. An abundant literature on diffusion data of various species in $\mathrm{Ni}$ and Ni-based alloys exists.

In a previous study [13], we characterized the deformation modes of the alloy by means of tensile tests over a wide range of thermo-mechanical conditions. Typical true stress-true strain curves obtained in a previous study for different temperatures of test on aged alloy 718 are plotted on Fig. 1.

The numerous tensile tests performed on alloy 718 allowed to draw accurately the occurrence domain of PLC serrations in the strain-rate - inverse temperature plane. Additionally this domain could be shared between normal and inverse behavior for the evolution of the critical strain to the onset of PLC effect. These domains are considered as respectively "low temperature" and "high temperature" of PLC effect. From this map, various methods were employed to calculate the apparent activation energies of the mechanisms involved in both normal and inverse PLC areas. A detailed description of the results and of the methods used to evaluate the apparent activation energies is the object of a previous publication [13].

Representative values of apparent activation energies obtained in this study are approximately $80-100 \mathrm{~kJ} / \mathrm{mol}$ for the low temperature (normal) PLC effect, and 200$280 \mathrm{~kJ} / \mathrm{mol}$ for the high temperature (inverse) PLC effect, which is consistent with previous studies on the alloy [9, $11,12]$. Thus, it was concluded that in the low temperature domain, the atomic element involved in the dynamic interaction with dislocations is free carbon, while in the high temperature PLC domain the element responsible for the flow instabilities is a substitutional one. Nevertheless, the scattering of the existing diffusion data of substitutional elements as well as of the apparent activation energies do not allow to conclude on the exact nature of the atomic element responsible for this temperature domain of the PLC effect in alloy 718 .
The present paper reports results from a mechanical spectroscopy study (internal friction) conducted on alloy 718 and various alloys with related chemical composition. This technique provides information on defects mobility in the alloy, such as point defects, dislocations or grain boundaries in the anelastic deformation domain $[14,15]$. As these defects are also those involved in plasticity, measurements of the anelastic contribution to deformation can be very useful in better understanding the interaction mechanisms as well as the atomic elements involved in the dynamic strain ageing phenomena during plastic deformation of the alloy.

\section{Materials}

Four different alloys have been used in this study: alloy 718 (hereafter referred as 718), a model alloy 718like but without $\mathrm{Nb}(718-\mathrm{Nb})$, a model alloy 718-like enriched in Mo $(718+\mathrm{Mo})$, and alloy $625+(625+)$. The chemical composition of the alloys as determined by Glow Discharge Mass Spectrometry (GDMS) is given in Table 1.

Alloy 718 used in the present study was provisioned in the form of single coil of rolled thin strip $(0.64 \mathrm{~mm}$ thick) in the solutionized state. The alloy exhibited an homogeneous equiaxed grain structure with mean grain size around 15 to $20 \mu \mathrm{m}$. Alloy $718-\mathrm{Nb}$ was provisioned as an hot forged ingot and required subsequent cold work and heat treatments to obtain a microstructure comparable with 718. The final grain size of the alloy is $15-30 \mu \mathrm{m}$. Alloy $718+$ Mo in the as-received state is a hot rolled plate of $6 \mathrm{~mm}$ thick. The alloy has been solutionized 1 hour dwell at $1060^{\circ} \mathrm{C}$ followed by fast quenching to remove the intergranular delta phase. The grain size is about 20 to $40 \mu \mathrm{m}$. Alloy $625+$ is a cold work plate of $4 \mathrm{~mm}$ thick which was solutionized. The grain size is about 15 to $30 \mu \mathrm{m}$.

Additionally an ageing heat treatment currently used for industrial application of alloy 718 has been applied to alloys $718,718+$ Mo and $625+$. Such treatment results mainly in the precipitation of hardening $\mathrm{Ni}_{3} \mathrm{Nb} \gamma^{\prime \prime}$-phase. 
Table 1. Chemical composition of the different alloys of the study (Wt \%).

\begin{tabular}{|l|l|l|l|l|l|l|l|l|l|l|l|}
\hline Element & Ni & Fe & Cr & Nb & Ti & Al & Mo & Mn & Si & Co & C \\
\hline Alloy 718 & bal. & 18.30 & 18.39 & 4.94 & 0.95 & 0.56 & 3.0 & 0.06 & 0.04 & 0.02 & 0.033 \\
\hline Alloy 718-Nb & bal. & 18.47 & 17.92 & $<0.001$ & 1.01 & 0.52 & 2.92 & 0.12 & 0.06 & 0.044 & 0.06 \\
\hline Alloy 718+Mo & bal. & 14.44 & 18.6 & 5.13 & 1.01 & 0.53 & 4.44 & 0.1 & 0.12 & 0.03 & 0.03 \\
\hline Alloy 625+ & bal. & 3.60 & 21 & 3.5 & 1.5 & 0.2 & 8.0 & 0.1 & 0.05 & 0.02 & 0.03 \\
\hline
\end{tabular}

This ageing treatment consists in two 8 hours dwells, respectively at temperatures $720^{\circ} \mathrm{C}$ and $620^{\circ} \mathrm{C}$ for alloys 718 and $718+\mathrm{Mo}$, and $735^{\circ} \mathrm{C}$ and $630^{\circ} \mathrm{C}$ for alloy $625+$. It is worth noting that treatment was not applied to alloy $718-\mathrm{Nb}$ which does not contain $\mathrm{Nb}$ and thus cannot form $\gamma^{\prime \prime}$ precipitates.

IF specimens with gage dimensions $50 \times 5 \mathrm{~mm}^{2}$ were cut either by disc saw machining (for alloy 718) or by wire electro-erosion (for the three other alloys). All samples surfaces were mechanically polished with $\mathrm{SiC}$ paper on at least $0.1 \mathrm{~mm}$ in order to remove the machining heat affected zone and until final surface 1200 grade SiC paper before testing.

\section{Experimental methods}

\subsection{Internal friction tests}

The internal friction (IF) and dynamic modulus measurements have been carried out on a forced sub-resonant torsion pendulum, able to work as a function of frequency in the range 0.01 to $3 \mathrm{~Hz}$ as well as to work as a function of temperature in the range 150 to $1100 \mathrm{~K}$, both with an excellent resolution [16]. In this study, the measures have been made as a function of temperature. The samples were tested at two frequencies per decade, during the heating of the sample at $1 \mathrm{~K} / \mathrm{min}$ and under a vacuum of about 1 to $2 \times 10^{-6} \mathrm{mbar}$, with initial oscillation deformation amplitude at room temperature of $1.0 \times 10^{-5}$. Temperature measures were made by mean of a K thermocouple located at the basis of the IF sample.

\subsection{Analysis method}

The tested samples spectra all received the same postanalysis which method is described in the following part and theoretically developed in [14]. The example proposed in this part concerns aged alloy $625+$. Typical spectra obtained after IF measurements appear on Fig. 2.

\subsubsection{Subtraction of the high temperature background}

The main point of the analysis of an IF spectrum is to subtract the high temperature background (HTB). The HTB is a phenomenon appearing for temperature of the order of 0.6 to $0.7 \mathrm{~T}_{\mathrm{m}}$ which is observed in many materials.

This is a thermally activated phenomenon that can be described as a relaxation process which characteristic time is very distributed and having a relaxation strength which is extremely high.

The HTB can be approximated by considering its low temperature beginning of peak by a standard relaxation

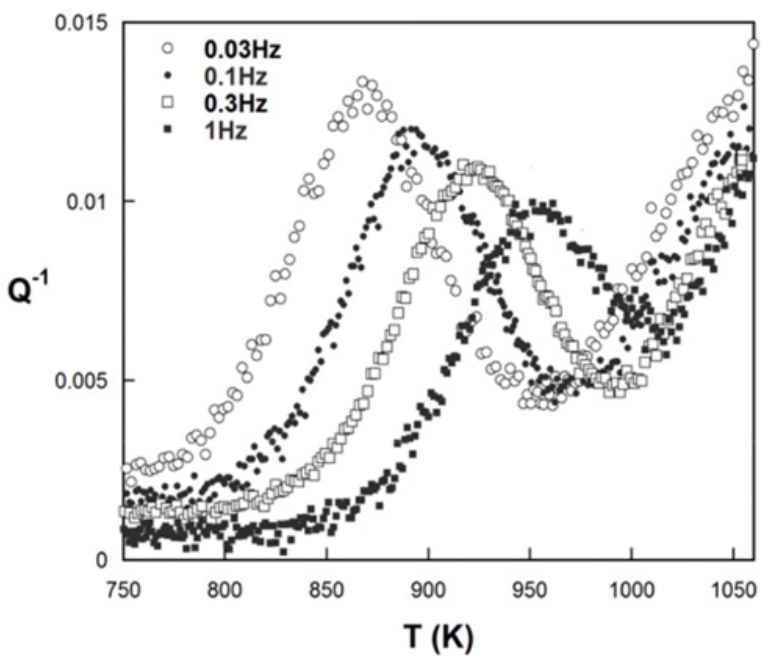

Figure 2. IF spectra obtained for alloy $625+$ at four frequencies varying from $1 \mathrm{~Hz}$ to $0.03 \mathrm{~Hz}$.

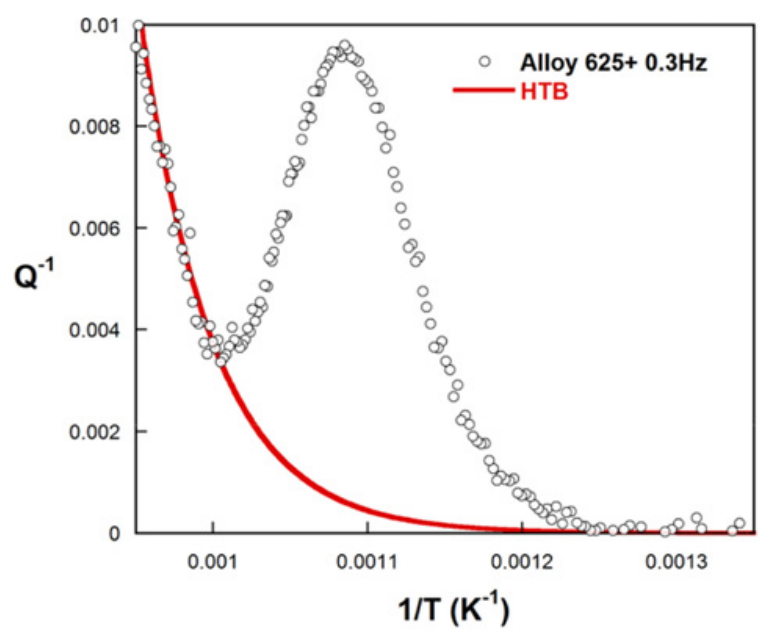

Figure 3. Determination of the high temperature background of a spectrum, example of alloy $625+$, for a frequency $0.3 \mathrm{~Hz}$.

peak, which means having an exponential envelope. The HTB of an IF spectrum is represented on Fig. 3.

The subtraction of the HTB from the spectrum leads to obtain a peak which is more symmetrical, modifying as well the position of the maximum of the peak in temperature, impacting by the way the activation energy calculation. The appearance of IF peaks after subtraction of the HTB is represented on Fig. 4.

On these corrected peaks different analysis have to be made in order to obtain the pertinent information allowing to conclude on the kind of defect and the mechanism implied in the relaxation process studied. 


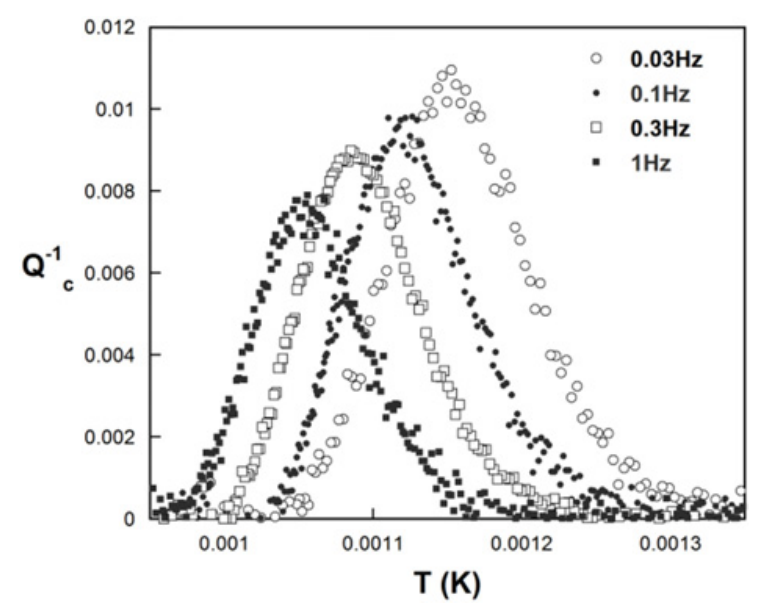

Figure 4. Corrected IF spectra for alloy $625+$ for frequencies varying from $1 \mathrm{~Hz}$ to $0.03 \mathrm{~Hz}$.

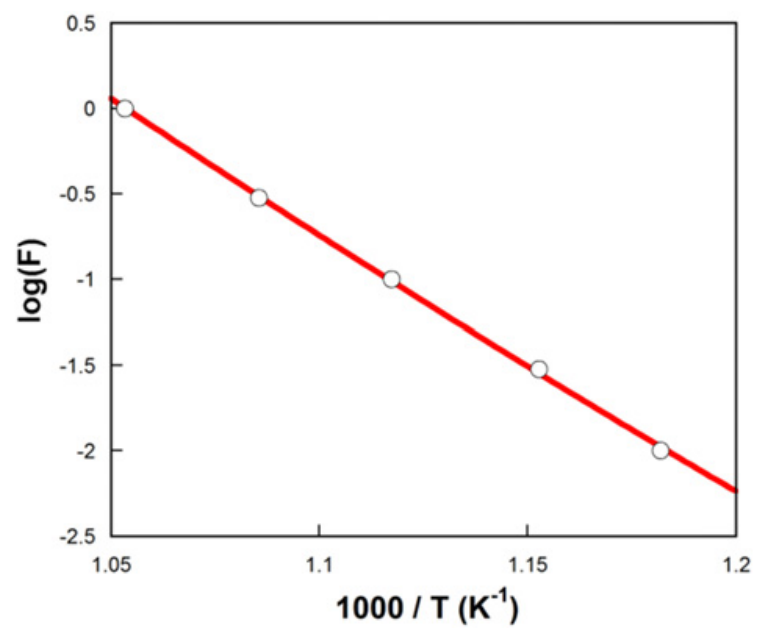

Figure 5. Plot of $\log (F)=f\left(\frac{1}{T_{p}}\right)$ or activation energy determination of the relaxation process generating the studied IF peak in alloy $625+$.

\subsubsection{Determination of the activation energy}

From the determination on the corrected spectra of the temperature of the peak maximum, $\left(T_{p}\right)_{F}$, or each solicitation frequency, $\mathrm{F}$, it is possible to determine the activation energy of the relaxation process implied in the apparition of the peak. Plotting $\log (F)=f\left(\frac{1}{T_{p}}\right)$ enerates a straight line corresponding to the Eq. (1).

$$
\log (F)=-\log \left(2 \pi \cdot \tau_{0}\right)-\frac{H_{a c t}}{2,3026 \cdot k} \cdot \frac{1}{T} 1 .
$$

The corresponding plot for alloy $625+$ is reported on Fig. 5. The slope of the linear fitting of the curve with an excellent correlation coefficient gives access to the activation energy of the relaxation process involved in the occurrence of the IF peak.

\subsubsection{Determination of the peak width}

The width of the peak is an indication of the distribution of relaxation times of the studied process. This is the reason why the peak shapes obtained in this study have

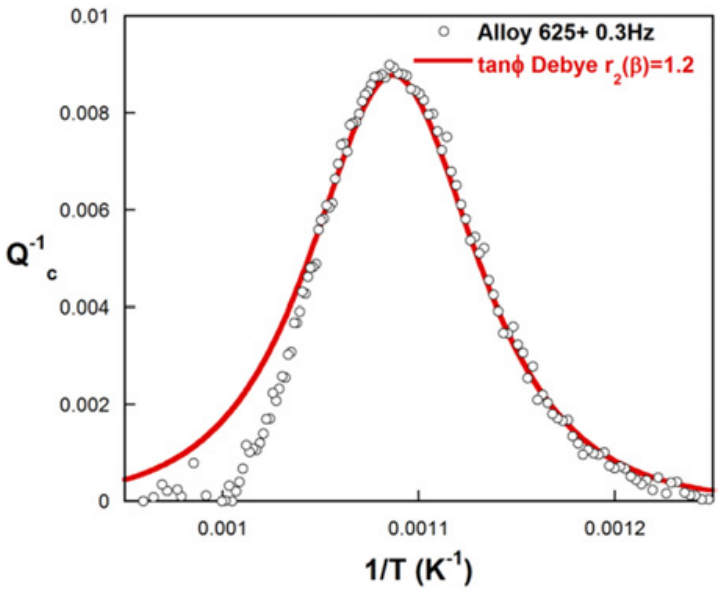

Figure 6. Fit of the corrected IF peak of alloy $625+$ for a frequency of $1 \mathrm{~Hz}$ by a Debye peak with a broadening factor of $r_{2}(\beta)=1.2$.

been compared to ideal Debye peaks, and their deviation to ideality quantified. In order to do so, it is necessary to compare the experimental corrected peak with theoretical peak for the same relative width [14], which is given by Eq. (2).

$Q^{-1}=\tan \phi=\tan \phi_{\max } \cdot \cosh { }^{-1}\left[\frac{H_{a c t}}{r_{2}(\beta) \cdot k} \cdot\left(\frac{1}{T}-\frac{1}{T_{p}}\right)\right] 2$.

In which $\tan \phi_{\max }$ the maximum height of the peak and $T_{p}$ he temperature of this maximum, $H_{a c t}$ is the activation energy determined by the previous method, $\mathrm{k}$ is the Boltzmann constant, and $r_{2}(\beta)$ is the broadening factor of the peak.

$r_{2}(\beta)$ is the variable quantity permitting to fit the experimental curve with the theoretical one: its value gives information about the dispersion of characteristic relaxation time, so about the type of mechanism involved in the relaxation process. Concerning the values of $r_{2}(\beta)$, it is generally considered that when $r_{2}(\beta)$ lies between 1 and 1.6, the relaxation process is linked to point defect and elastic dipoles reorientation, while above this value, the process may involve dislocations deforming around an equilibrium position, or grain boundaries.

An example of such a fitting of an experimental IF peak by enlarged Debye peaks appears on Fig. 6.

The broadening factor chosen to fit this spectrum is $r_{2}(\beta)=1.2$, which corresponds to a relaxation process due to point defects.

\section{Results and interpretation}

\subsection{Internal friction tests}

A first aspect that has to be mentioned is that all the tested alloys exhibit only one IF peak in the studied temperature range $(298-1100 \mathrm{~K})$. The results of the IF tests, in terms of activation energies, relaxation times, and IF peak broadening factor appear in Table 2 .

These first observations on different alloys and metallurgical conditions give the following information: 
Table 2. Summary of the IF spectra analysis for the different alloys of the study.

\begin{tabular}{|l|l|l|l|}
\hline Alloy & $\mathbf{Q}_{\text {act }}(\mathbf{k J} / \mathbf{m o l})$ & $\tau_{\mathbf{0}}(\mathbf{s})$ & $\mathbf{r}_{\mathbf{2}}(\beta)$ \\
\hline Alloy 718 & 292 & $9.8 \times 10^{-16}$ & 1.2 \\
\hline Alloy 718-Nb & 300 & $4.9 \times 10^{-16}$ & 1.3 \\
\hline Alloy 718+Mo & 291 & $1.0 \times 10^{-17}$ & 1.3 \\
\hline Alloy 625+ & 294 & $3.0 \times 10^{-18}$ & 1.2 \\
\hline
\end{tabular}

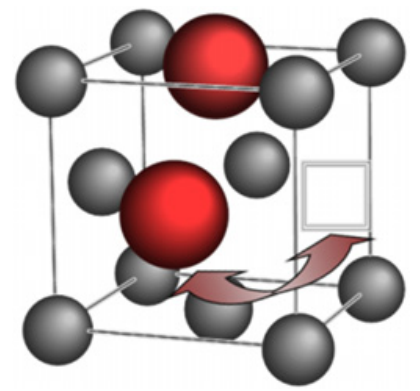

Figure 7. Relaxation mechanism of the Zener peak: reorientation of a substitutional dipole by exchange of one substitutional with a vacancy under cyclic applied stress.

the broadening factors of the IF peaks are between 1.2 and 1.3, which leads to conclude that the IF peaks observed are linked to point defects. The activation energies calculated from the peaks at different frequencies are close to the diffusion energies of substitutional solute atoms in $\mathrm{Ni}$ and Ni-base alloys, between 290 and $300 \mathrm{~kJ} / \mathrm{mol}$.

What can be inferred from these first results is that the peak observed and analyzed in all four alloys is a Zener peak $[15,17]$ caused by the reorientation of elastic dipoles constituted by pairs of substitutional atoms and a vacancy closest neighbor, as explained in Fig. 7. An important point is that, for a Zener peak, the relaxation strength $\Delta$ (which is linked to the height of the peak) is related to the solute concentration of the substitutional element involved in its apparition. This is true for dilute solid solutions, as well as for concentrated solid solutions $[18,19]$. Which means that, in this study, the comparison of the IF peak heights of different alloys can provide important information on the nature of the element involved in the relaxation process.

\subsection{Comparisons of IF peak heights}

A first comparison can be made upon alloys exhibiting different solute $\mathrm{Nb}$ contents. Three different specimens have been considered: alloy 718 in the standard (heat treated) state, the same alloy in the solutionized state and the alloy $718-\mathrm{Nb}$. IF results show that the three specimens exhibit the same Zener peak in terms of temperature and frequency behavior. The peaks shown by alloys in solid solution states (718 and 718-Nb) are exactly superimposed while the heat treated 718 alloy shows a slightly higher peak. These results clearly demonstrate that the solute concentration on $\mathrm{Nb}$ does not influences the IF peak, that is this peak cannot be related to the presence of $\mathrm{Nb}$ atoms. Similarly the influence of $\mathrm{Al}$ and $\mathrm{Ti}$ species can also be disregarded since their solute content strongly varies during the precipitation heat treatment.

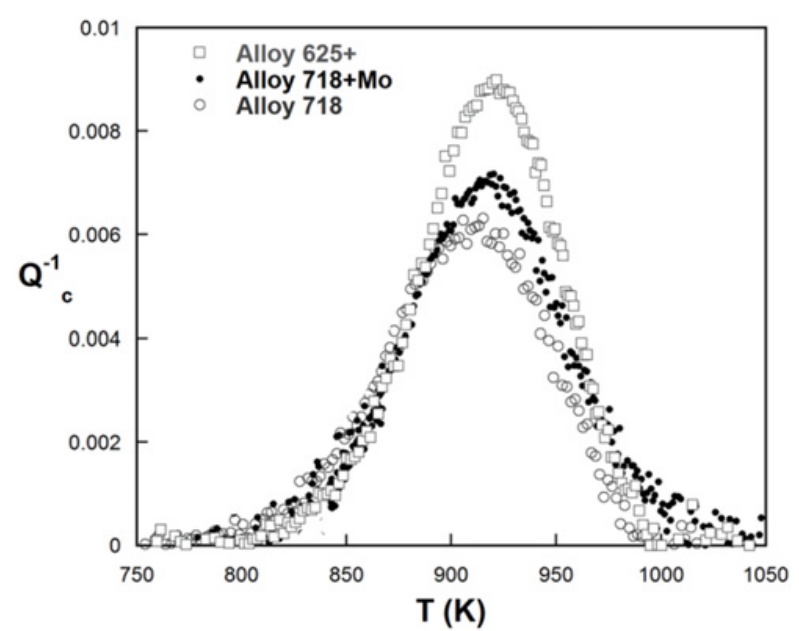

Figure 8. Comparison of the peak heights at $\mathrm{F}=0.3 \mathrm{~Hz}$ of alloys 718 (3wt\%Mo), 718+Mo (4.4wt\%Mo) and 625+ (8wt\%Mo).

The last comparison of alloys providing important information is the one between aged alloys, containing different levels of Mo, namely: alloy 718, alloy 718+Mo and alloy $625+$, with respectively $3 \mathrm{wt} \%, 4.4 \mathrm{wt} \%$ and $8 \mathrm{wt} \%$ Mo content, as mentioned in Table 1 . The purpose is to evaluate if the Mo content of the alloy has an influence on the relaxation strength, $\Delta$, by comparison of the height of the IF peaks in relation with the composition. This comparison is the object of Fig. 8. The height of the IF peak increases for the different alloys 718, 718+Mo and $625+$.

When considering the chemical compositions of these alloys (Table 1), it appears clearly that the only alloying element, which varies in a coherent way with the height of the peak between the three alloys, is Mo.

This study by means of mechanical spectroscopy shows that all the tested alloys exhibit an IF peak related to Zener relaxation. The comparison of the result obtained for the alloys with different chemical composition shows that $\mathrm{Nb}$ is not involved in this relaxation but highlights the effect of Mo.

\section{Discussion}

The tensile tests carried out on wide ranges of temperatures and strain rates in a previous study [13] permitted to distinguish between two domains of different types of PLC effects: a low temperature PLC effect, and a high temperature PLC effect. It is commonly agreed that for low temperature domain, the origin of flow instabilities is the interaction between mobile dislocations and $\mathrm{C}$ atoms. By contrast, for high temperatures PLC effect, different substitutional elements have been proposed, without providing very convincing arguments. Indeed, the last proposition appearing in literature was that $\mathrm{Nb}$ is the element responsible for high temperature PLC effect [12]. Nevertheless, we recently carried out tensile tests on alloy $\mathrm{B}$ (without $\mathrm{Nb}$ ), in the high temperature PLC domain $\left(650^{\circ} \mathrm{C}-10^{-4} \mathrm{~s}^{-1}\right)$ which exhibited plastic instabilities. This means that $\mathrm{Nb}$ cannot be considered as the only element involved in the occurrence of flow instabilities, or may not be involved at all. 
The internal friction tests tend to prove that the IF peaks observed in alloy 718 and various related alloys are due to the presence of Mo. This interaction at an atomic scale has a strong impact on the damping capacity of the alloy in certain temperature domain. Moreover, the activation energy associated to the IF peak measured in this study seems to agree pretty well with Mo jump energy estimated by ab-initio calculations (Max et al. to be published). The fact that Mo seems to be the only substitutional element developing such an interaction in the Ni matrix of the alloy is a relevant fact: its influence is probably not a second order parameter when it comes to understanding the mechanical behavior of the alloy.

Although the deformation modes are different between IF and tensile testing, as in one case it implies the dynamic interaction of alloying elements with mobile dislocation, and in the other case it concerns the short range diffusion of point defects between different equilibrium positions, IF results provide important information to understand the interactions occurring during plastic deformation. Indeed, the capacity of Mo to strongly interact with the host lattice can be related with its ability to interact with mobile dislocations.

Finally, the implication of Mo in the occurrence of DSA and PLC effect in alloy 718 at high temperature, which has been until now ruled out by most of the authors on the subject, should be taken into consideration. The tensile behavior and the mechanical response of this alloy as a function of Mo content should be carefully studied.

\section{Conclusion}

The mechanical spectroscopy study carried out on alloy 718 and various related alloys brings new elements to the understanding of solid solution interaction of some alloying atomic species. The internal friction spectra of all alloys characterized in this study present a Zener relaxation peak. The mechanism involved is the reorientation of dipoles constituted by substitutional atoms pairs by atomic jump of a substitutional to a close neighbor site. The analysis of the spectra shows that the substitutional element involved in this relaxation process is the same for all alloys. The comparative analysis of the relaxation intensities on the alloys with monitored changes in chemical composition proves that Niobium is not the element responsible for this process. By contrast, the relaxation process intensity seems to be directly linked to the solid solution Molybdenum content of the alloy, which proves that it is the substitutional element involved in the Zener relaxation mechanism in these alloys.

This interaction between Molybdenum atoms associated as dipoles and the Ni-matrix has a strong impact on the internal damping phenomenon of the alloy. This leads to consider that the interaction between Molybdenum and other defects in alloy 718 should be of a relevant order of magnitude. The impact of this element on the deformation modes of alloy 718 , often considered as a second order parameter, may not be marginal. In the case of plastic deformation, the effect of Molybdenum in the dynamic interactions with mobile dislocations in the high temperatures domain should not be excluded; particularly its influence on the occurrence and the characteristics of PLC effect may deserve some detailed studies.

J. San Juan and M.L. Nó, thank the financial support from the Consolidated Research Group IT-10-310 from the Education Department and the project ETORTEK ACTIMAT from the Industry Department of Basque Government.

\section{References}

[1] X. Pang, D. Dwyer, M. Gao, P. Valerio, R. Wei, Scripta Met. Mat. 31, 345 (1994)

[2] C. F. Miller, G. W. Simmons, R. P. Wei, Scripta Mat. 42, 227 (2000)

[3] C. F. Miller, G. W. Simmons, R. P. Wei, Scripta Mat. 44, 2405 (2001)

[4] C. T. Sims, Superalloys 1984, 399 (1984)

[5] R. MacKay, M. Nathal, D. Pearson, Met. Trans. A 21, 381 (1990)

[6] H. Eiselstein, D. Tillack, International Symposium on Metallurgy and Applications of Superalloys, 718, 625, (1991)

[7] L. Fournier, D. Delafosse, T. Magnin, Mat. Sci. Eng. A316, 166 (2001)

[8] V. Garat, J.-M. Cloué, D. Poquillon, E. Andrieu, J. Nucl. Mat. 375, 95 (2008)

[9] R. W. Hayes, Acta Met. 31, 365 (1983)

[10] W. Chen, M. C. Chaturvedi, Mat. Sci. Eng. A229, 163 (1997)

[11] C. L. Hale, W. S. Rollings, M. L. Weaver, Mat. Sci. Eng. A300, 153 (2001)

[12] S. A. Nalawade, M. Sundararaman, R. Kishore, J. G. Shah, Scripta Mat. 59, 991 (2008)

[13] B. Max, B. Viguier, E. Andrieu, J.-M. Cloué, Met. Mat. Trans. A, to be published

[14] J. San Juan, Mat. Sci. For., 366, 32, (2001)

[15] A. Nowick, B. Berry, Anelastic relaxation in crystalline solids. Academic Press, New York (1972)

[16] I. Gutierrez-Urrutia, M. L. N, E. Carreo-Morelli, B. Guisolan, R. Schaller, J. San Juan, Mat. Sci. Eng. A 370, 435 (2004)

[17] C. Zener, Phys. Rev. 71, 34 (1947)

[18] A. Nowick, D. Seraphim, Acta Met. 9, 40 (1961)

[19] D. Seraphim, A. Nowick, Acta Met. 9, 85 (1961) 